Pure and Applied Mathematics Quarterly

Volume 1, Number 2

(Special Issue: In memory of

Armand Borel, part 1 of 3)

$341-367,2005$

\title{
Converse Theorems, Functoriality, and Applications
}

\author{
J.W. Cogdell
}

Converse Theorems traditionally have provided a way to characterize Dirichlet series associated to modular forms in terms of their analytic properties. The prototypical Converse Theorem was due to Hamburger who characterized the Riemann zeta function in terms of its analytic properties [23]. More familiar may be the Converse Theorems of Hecke and Weil. Hecke first proved that Dirichlet series associated to modular forms enjoyed "nice" analytic properties and then proved "Conversely" that these analytic properties in fact characterized modular Dirichlet series [26]. Weil extended this Converse Theorem to Dirichlet series associated to modular forms with level [62]. In their modern formulation, Converse Theorems are stated in terms of automorphic representations instead of modular forms. This was first done by Jacquet and Langlands for $G L_{2}$ [31]. For $G L_{n}$, Jacquet, Piatetski-Shapiro, and Shalika have proved that the $L$-functions associated to automorphic representations have nice analytic properties similar to those of Hecke [32] (see also [15, 9]). The relevant "nice" properties are: analytic continuation, boundedness in vertical strips, and functional equation. Converse Theorems in this context invert this process and give a criterion for automorphy, or modularity, in terms of these $L$-functions being "nice" [31, 32, 13, 9].

The first application of a Converse Theorem that might come to mind is to the question of modularity of arithmetic or geometric objects. To use the Converse Theorem in this context one must first be able to prove that the $L$ functions of these arithmetic/geometric objects are "nice". However, essentially the only way to show that an $L$-function is nice is to have it associated to an automorphic form already! So, on second thought, as a direct tool for establishing modularity the Converse Theorem seems a bit lacking. Instead, the most natural direct application of the Converse Theorem is to Functoriality, in this case the

Received April 24, 2005. 
transfer of automorphic representations from some group $G$ to $G L_{n}$. Recently this approach to Functoriality via the Converse Theorem has proved successful and many new cases of Functoriality have been established (see Section 2.4 below).

Further applications of the Converse Theorem come about through applications of these cases of Functoriality. As a meta-application, Functoriality itself is a manifestation of Langlands' vision of a non-abelian class field theory, so that proving explicit cases of Functoriality gives direct evidence of this vision. On the other hand, one must not lose sight of the fact that automorphic forms, like classical modular forms, can be formidable tools in analytic number theory. As an application of some of these recent cases of Functoriality, Kim and Shahidi have established the best known general bounds towards the Ramanujan conjecture for $G L_{2}$ (see Section 3.1 below). Applying these bounds in the case of $G L_{2}$ over a totally real field was an ingredient that we used (with Piatetski-Shapiro and Sarnak) in proving a subconvexity result for twisted Hilbert modular $L$-functions, which in turn was the crucial estimate in our confirmation of the last remaining case of Hilbert's eleventh problem (see Section 3.2 below). Of course, one should never lose sight of the question of modularity and one can reasonably ask if the Converse theorem played any role in the recent spectacular cases of modularity: modularity of elliptic curves over $\mathbb{Q}$ by Wiles, et.al., and the modularity of global Galois representations in the function field setting by Lafforgue. The answer is (luckily) yes, and we will discuss this briefly at the end.

This article is based on the Whittemore Lectures that I gave at Yale in the Fall of 2001. In those lectures I presented an overview of this circle of ideas. In the first lecture I discussed the classical results of Hecke and Weil, the problems they were interested in at the time, and then the modern formulations of the Converse Theorem. The second lecture I devoted to the "what" and "why" of Functoriality as well as the use of the Converse Theorem in establishing new cases of Functoriality. In the final lecture I turned to applications of these results to various questions in number theory including their relation to Hilbert's eleventh problem and to general questions of "modularity". As the Whittemore Lectures were to present an introduction of this material to a general mathematical audience, they were somewhat colloquial in tone and purposefully avoided many technical details. Since I hope that this article can fulfill a similar purpose, I have retained the tone of the lectures. This account is by no means encyclopedic or even complete and I apologize in advance to all those who have contributed but whose contributions I have failed to give complete coverage. More details can be found in the research papers listed in the bibliography.

It is an honor to be able to present my Whittemore Lectures in this volume dedicated to Borel. I was quite lucky to be a graduate student at Yale when Borel 
spent the Fall of 1978 there. That year Borel gave the Whittemore Lectures, probably the first series that I attended, as well as a graduate course on "Some Topics in the Analytic theory of Automorphic Forms". To this day, the notes that I took in Borel's course are my primary reference for the analytic theory of automorphic forms, including the analytic continuation of rank 1 Eisenstein series and the associated spectral decomposition of $L^{2}$. It was a truly formative experience in my career, both in terms of the actual mathematics learned and possibly more importantly in terms of the conveying a sense of respect for the mathematics.

\section{Converse Theorems}

As always, we begin with Hecke and the theory of classical modular forms [28]. If we let $\Gamma=S L_{2}(\mathbb{Z})$ and $\mathfrak{H}=\{z=x+i y \mid y>0\}$ then a modular form of weight $k \geq 2$ for $\Gamma$ is a holomorphic function $f: \mathfrak{H} \rightarrow \mathbb{C}$ such that

1. $f\left(\frac{a z+b}{c z+d}\right)=(c z+d)^{k} f(z)$ for all $\left(\begin{array}{ll}a & b \\ c & d\end{array}\right) \in \Gamma$;

2. $f$ is "holomorphic at $\infty$ ".

For this last condition, note that $\Gamma \backslash \mathfrak{H} \simeq \mathbb{P}^{1}(\mathbb{C})-\{\infty\}$. Let $M_{k}(\Gamma)$ denote the (finite dimensional) space of all such forms of weight $k$.

These functions have often played a key role in arithmetic questions, so much so that Eichler purportedly stated that there are five fundamental operations in arithmetic: addition, subtraction, multiplication, division, and modular forms. expansion

Since $T=\left(\begin{array}{ll}1 & 1 \\ 0 & 1\end{array}\right) \in \Gamma$, we see $f(z+1)=f(z)$ and so we have a Fourier

$$
f(z)=\sum_{n=0}^{\infty} a_{n} e^{2 \pi i n z}
$$

beginning with $n=0$ since $f(z)$ is holomorphic at $\infty . f(z)$ is a cusp form if

$$
f(z)=\sum_{n=1}^{\infty} a_{n} e^{2 \pi i n z},
$$

i.e., if $f$ vanishes at the cusp at $\infty$. The space of cusp forms of weight $k$ is denoted $S_{k}(\Gamma)$ 
Hecke associated to $f(z)$ an $L$-function

$$
L(s, f)=\sum_{n=1}^{\infty} \frac{a_{n}}{n^{s}} \quad \operatorname{Re}(s)>\frac{k}{2}+1
$$

and introduced an algebra of operators (the original Hecke algebra) $\mathcal{H}=\left\langle T_{p}\right\rangle$, generated by the Hecke operators $T_{p}$ indexed by primes, such that $L(s, f)$ has an Euler product expansion iff $f$ is an eigenfunction of all Hecke operators, and then

$$
L(s, f)=\prod_{p}\left(1-a_{p} p^{-s}+p^{k-1} p^{-2 s}\right)^{-1} .
$$

If $f \in S_{k}(\Gamma)$ then Hecke showed that these $L$-functions were nice. If we set

$$
\Lambda(s, f)=(2 \pi)^{-2} \Gamma(s) L(s, f)
$$

then

(i) $\Lambda(s, f)$ extends to an entire function of $s$;

(ii) $\Lambda(s, f)$ is bounded in vertical strips;

(iii) $\Lambda(s, f)$ satisfies the functional equation

$$
\Lambda(s, f)=i^{k} \Lambda(k-s, f) .
$$

These nice properties of $\Lambda(s, f)$ followed from the modularity of $f$ via the Mellin transform

$$
\Lambda(s, f)=\int_{0}^{\infty} f(i y) y^{s} d^{\times} y .
$$

In particular, the functional equation is equivalent to the transformation law $f(S z)=f(-1 / z)=z^{k} f(z)$ for $S=\left(\begin{array}{cc}0 & -1 \\ 1 & 0\end{array}\right) \in \Gamma$.

Moreover, Hecke realized that he could establish a converse to this since the Mellin transform is invertible [26, 27]. Namely, if the Dirichlet series

$$
D(s)=\sum_{n=1}^{\infty} \frac{a_{n}}{n^{s}} \quad \operatorname{Re}(s)>>0
$$

is nice, i.e.,

$$
\Lambda(s)=(2 \pi)^{-s} \Gamma(s) D(s)
$$


extends to an entire function, is bounded in vertical strips, and satisfies

$$
\Lambda(s)=i^{k} \Lambda(k-s)
$$

then

$$
f(z)=\sum_{n=1}^{\infty} a_{n} e^{2 \pi i n z} \in S_{k}(\Gamma)
$$

Once again,

$$
f(i y)=\sum_{n=1}^{\infty} a_{n} e^{-2 \pi n y}=\int_{\operatorname{Re}(s)=c} \Lambda(s) y^{-s} d s
$$

and the modular transformation for $f(z)$ under $S$ comes from the functional equation for $\Lambda(s)$.

Weil proved a similar converse theorem for modular forms $f \in S_{k}(\Gamma)$ for $\Gamma=\Gamma_{0}(N)[62]$. Note that now $S=\left(\begin{array}{cc}0-1 \\ 1 & 0\end{array}\right)$ is no longer an element of $\Gamma$. Instead of requiring that only $\Lambda(s)=(2 \pi)^{-s} \Gamma(s) D(s)$ be nice for $D(s)=\sum a_{n} n^{-s}$ he had to require that the twisted $L$-function

$$
\Lambda(s, \chi)=(2 \pi)^{-s} \Gamma(s) \sum_{n=1}^{\infty} \frac{\chi(n) a_{n}}{n^{s}}
$$

is appropriately nice for (essentially) all Dirichlet characters $\chi$ of conductor prime to $N$ to conclude that $f(z)=\sum a_{n} e^{2 \pi i n z} \in S_{k}(\Gamma)$ and that $D(s)=L(s, f)$.

What questions were Hecke and Weil interested in?

Hecke [26, 27]: Hamburger (1921-22) had proved that the Riemann zeta function $\zeta(s)$ was completely characterized by its analytic properties, particularly the functional equation [23]. Hecke was interested in such results for other fields $K$. The idea was to attach to the Dedekind zeta function $\zeta_{K}(s)$ a modular form and then use the modular structure (finite dimensionality, Hecke operators, etc.) to obtain the characterization. This he could do for quadratic imaginary $K$.

Weil [62]: Weil was looking at something more conjectural. If we let $E$ : $y^{2}=x^{3}+a x+b$ be an elliptic curve over $\mathbb{Q}$, then $E$ has associated to it an $L$-function built as an Euler product

$$
L(E, s)=\left(\prod_{p \mid 2 \Delta} \cdots\right) \prod_{p \nmid 2 \Delta}\left(1-a_{p} p^{-s}+p^{1-2 s}\right)^{-1} \quad \operatorname{Re}(s)>\frac{3}{2}
$$

where $a_{p}=p-\left|\bar{E}\left(\mathbb{F}_{p}\right)\right|+1$ is related to the number of points on $\bar{E}$, the reduction of $E \bmod p$. It had been conjectured by Taniyama and Shimura that $E$ should be 
modular, i.e., associated to a cusp form of weight 2 . Since $L(E, s)$ and the twisted $L$-functions $L(E, s, \chi)$ were expected to be nice then Weil's Converse Theorem would give an explicit connection with modular forms.

So both Hecke and Weil were interested in questions of modularity - attaching modular forms to arithmetic objects - mediated by L-functions.

Note 1: Of course, we now know that all elliptic curves over $\mathbb{Q}$ are modular: Wiles, Taylor, Breuil, Conrad, Diamond [63, 6].

Note 2: As an example of why these $L$-functions of arithmetic objects are interesting, recall the Birch and Swinnerton-Dyer Conjecture [64] for elliptic curves: $L(E, s)$ is built out of purely local information yet extends to all $\mathbb{C}$ and has a functional equation relating $L(E, s)$ and $L(E, 2-s)$. The central point is $s=1$ and according to Birch and Swinnerton-Dyer this central point carries very interesting global information, the easiest example being that the order of vanishing of $L(E, s)$ at $s=1$ should equal the rank of the group of rational points $E(\mathbb{Q})$.

The modern formulation of the Converse Theorem are connected with the modern formulation of modular forms: automorphic representations. For more details and references for the theory of automorphic representations and $L$-functions for $G L_{n}$, we refer the reader to [9].

Take $k$ a number field, say $k=\mathbb{Q}$ (a global function field would work just as well). The adele ring $\mathbb{A}=\mathbb{A}_{k}$ is the restricted product of all completions of $k$ : $\mathbb{A}_{k}=\prod^{\prime} k_{v}$

$$
\mathbb{A}_{\mathbb{Q}}=\mathbb{R} \times \prod_{p}^{\prime} \mathbb{Q}_{p}=\underset{S}{\lim }\left(\mathbb{R} \times \prod_{p \in S} \mathbb{Q}_{p} \times \prod_{p \notin S} \mathbb{Z}_{p}\right) .
$$

Then $k$ embeds diagonally in $\mathbb{A}$ as a canonical discrete sub-ring and $k \backslash \mathbb{A}$ is compact. Similarly, for $G L_{n}$ one can consider $G L_{n}(\mathbb{A})=\prod_{v}{ }^{\prime} G L_{n}\left(k_{v}\right)$ and $G L_{n}(k)$ embeds diagonally in $G L_{n}(\mathbb{A})$ as a canonical discrete subgroup with finite covolume (modulo the center). The space of $L^{2}$-automorphic forms on $G L_{n}(\mathbb{A})$, with a fixed behavior (by a unitary character $\omega$ ) under the center is simply $L^{2}\left(G L_{n}(k) \backslash G L_{n}(\mathbb{A}) ; \omega\right)$ and this affords a representation of $G L_{n}(\mathbb{A})$ by right translation. The space of cusp forms, denoted $L_{0}^{2}\left(G L_{n}(k) \backslash G L_{n}(\mathbb{A}) ; \omega\right)$, is a stable subspace. These are the automorphic forms which "vanish at all cusps" or whose "constant Fourier coefficients" are 0. A result of Gelfand and PiatetskiShapiro [22] tells us that the space of cusp forms decomposes discretely with finite multiplicities

$$
L_{0}^{2}\left(G L_{n}(k) \backslash G L_{n}(\mathbb{A}) ; \omega\right)=\oplus m_{\pi} V_{\pi}
$$

with $m_{\pi}<\infty$ (and in fact equal to 1 if non-zero). The components $\left(\pi, V_{\pi}\right)$ are the cuspidal automorphic representations. 
Note: If $f(z)$ is a classical modular form which is an eigenfunction for all the Hecke operators, then $f$ completely determines one of these components $\left(\pi, V_{\pi}\right)$ for $n=2$, i.e, determines and is determined by a cuspidal automorphic representation of $G L_{2}(\mathbb{A})$.

Just as we have the decomposition $G L_{n}(\mathbb{A})=\prod_{v}{ }^{\prime} G L_{n}\left(k_{v}\right)$ the irreducible cuspidal representations also factor $\pi \simeq \otimes_{v}^{\prime} \pi_{v}$ into a restricted tensor product of irreducible admissible unitary representations $\pi_{v}$ of $G L_{n}\left(k_{v}\right)$.

In the 1970-80's, Jacquet, Piatetski-Shapiro and Shalika, following Jacquet and Langlands, extended the work of Hecke to give a theory of $L$-functions for cuspidal automorphic representations of $G L_{n}(\mathbb{A})$ via integral representations. For each $\left(\pi, V_{\pi}\right)$ with $\pi \simeq \otimes_{v}^{\prime} \pi_{v}$ they associated

1. $\pi_{v} \mapsto L\left(s, \pi_{v}\right)$ a local Euler factor at $v ; L\left(s, \pi_{v}\right)=P_{v}\left(q_{v}^{-s}\right)^{-1}$ for $P_{v}$ a polynomial of degree $n$ with $P_{v}(0)=1$ at almost all finite places $v$ and for $v \mid \infty L\left(s, \pi_{v}\right)$ is an appropriate product of shifted $\Gamma$-functions of "degree $n$ ".

2. $\pi \mapsto L(s, \pi)=\prod_{v} L\left(s, \pi_{v}\right)$; an Euler product, convergent for $\operatorname{Re}(s)>>0$.

Similarly, by analogy with Weil, for $\pi^{\prime} \simeq \otimes^{\prime} \pi_{v}^{\prime}$ a cuspidal representation of $G L_{m}(\mathbb{A}), m \leq n$, they defined twisted $L$-functions

$$
L\left(s, \pi \times \pi^{\prime}\right)=\prod_{v} L\left(s, \pi_{v} \times \pi_{v}^{\prime}\right)
$$

which are Euler products of degree $n \cdot m$ (the analogue of $\Lambda(s, f, \chi)$ before) and they proved that these were nice:

(i) extend to entire functions of $s$ (as long as $m<n$ );

(ii) are bounded in vertical strips of finite width;

(iii) satisfy a functional equation

$$
L\left(s, \pi \times \pi^{\prime}\right)=\varepsilon\left(s, \pi \times \pi^{\prime}\right) L\left(1-s, \tilde{\pi} \times \tilde{\pi}^{\prime}\right)
$$

with the $\varepsilon$-factor a monomial and $\tilde{\pi}$ representing the contragredient (or dual) representation.

(To be honest, the method of integral representations currently can show boundedness in vertical strips only if $m=n$ or $m=n-1$ [15]. In the other cases we must rely on the work of Gelbart and Shahidi [21].) Again, as in the classical case, automorphic L-functions are nice. 
What shape does a Converse Theorem now take? One could begin with an arbitrary $L$-function as defined by an Euler product, but this does not exhibit enough structure. (If $L(s)$ is degree $n$ over $\mathbb{Q}$ is it to come from an automorphic form for $G L_{n}$ over $\mathbb{Q}$ or from $G L_{1}$ over a field $K$ with $(K: \mathbb{Q})=n$, or somewhere in between.) Instead we are a bit more specific. We begin with $\Pi=\otimes_{v}^{\prime} \Pi_{v}$ an irreducible admissible representation of $G L_{n}(\mathbb{A})$ thought of as a collection of local data that need not be coherent. (We do add one coherence condition: the central character $\omega_{\Pi}$ should be automorphic.) Then the local theory of Jacquet, Piatetski-Shapiro and Shalika [34] at least lets us formally define an $L$-function

$$
L(s, \Pi)=\prod_{v} L\left(s, \Pi_{v}\right) .
$$

So $\Pi$ encodes an $L$-function as an Euler product of degree $n$, but with some added structure. Moreover for $\pi^{\prime}$ now a cuspidal automorphic representation of $G L_{m}(\mathbb{A})$ we can similarly form a twisted $L$-function

$$
L\left(s, \Pi \times \pi^{\prime}\right)=\prod_{v} L\left(s, \Pi_{v} \times \pi_{v}^{\prime}\right)
$$

just as Weil did.

Converse Theorem I. [13] Suppose $L(s, \Pi)$ converges for $\operatorname{Re}(s)>>0$ and $L\left(s, \Pi \times \pi^{\prime}\right)$ is nice for all cuspidal $\pi^{\prime}$ for $G L_{m}(\mathbb{A})$ with $1 \leq m \leq n-1$. Then $\Pi$ is a cuspidal automorphic representation of $G L_{n}(\mathbb{A})$.

This is the analogue of Weil's result. Improving upon Weil we have:

Converse Theorem II. [14] Suppose $n \geq 3$ and $L\left(s, \Pi \times \pi^{\prime}\right)$ is nice for all cuspidal $\pi^{\prime}$ for $G L_{m}(\mathbb{A})$ with $1 \leq m \leq n-2$. Then $\Pi$ is a cuspidal automorphic representation of $G L_{n}(\mathbb{A})$.

Note: There are also more useful variants (as with Weil) which allow various types of restriction on the ramification of the twisting representations, but in exchange one loses control of the local components of $\Pi$ at a finite number of places.

The most one could hope for is the following.

Conjecture. [13] Suppose $L(s, \Pi \times \omega)$ is nice for all idele class characters $\omega$. Then there exists an automorphic representation $\Pi^{\prime}$ of $G L_{n}(\mathbb{A})$ such that $L(s, \Pi \times$ $\omega)=L\left(s, \Pi^{\prime} \times \omega\right)$ for all $\omega$.

In other words, the $L$-function you started with was automorphic, you had just parameterized it in the wrong way. This conjecture would be quite powerful: see Taylor's address in the 2002 ICM $[57,58]$. 
Who cares? The first thought is to Weil and his modularity. He didn't actually prove that $L(E, s)$ was modular, but he said that if you expect a degree $2 L$-function to be nice then it must be modular.

Degree $n L$-functions also arise in arithmetic and geometry. One rich family come from either complex or $\ell$-adic Galois representations

$$
\rho: \operatorname{Gal}(\bar{k} / k) \rightarrow G L_{n}(\mathbb{C}) \quad \text { or } \quad \rho: G a l(\bar{k} / k) \rightarrow G L_{n}\left(\overline{\mathbb{Q}}_{\ell}\right) .
$$

These have $L$-functions

$$
L(s, \rho)=\prod L_{v}(s, \rho)
$$

defined as Euler products convergent for $\operatorname{Re}(s)>>0$. For most places $v$ (unramified for $\rho$ ) we have

$$
L_{v}(s, \rho)=\operatorname{det}\left(1-\rho\left(\operatorname{Frob}_{v}\right) q_{v}^{-s}\right)^{-1}
$$

is a degree $n$ Euler factor. Since these and almost all other arithmetic or geometric $L$-functions are expected to be nice then these Galois representations should be modular. This is the meta-application of the Converse Theorems and we will discuss it more below.

But what about a real application?

\section{Functoriality}

\subsection{Langlands Conjectures}

(For more details and references, we refer the reader to [5] and to Chapter 10 of [8].) We begin with a specific case of modularity. One of the primary goals of number theory is to understand the group $\mathcal{G}_{\mathbb{Q}}=\operatorname{Gal}(\overline{\mathbb{Q}} / \mathbb{Q})$ or more generally $\mathcal{G}_{k}=\operatorname{Gal}(\bar{k} / k)$ since this group governs the arithmetic of $k$. One way to try to understand $\mathcal{G}_{k}$ is through understanding its finite dimensional representations

$$
\rho: \mathcal{G}_{k} \rightarrow G L_{n}(\mathbb{C}) .
$$

It was Artin who in 1930 attached to these representations an analytic invariant, now called that Artin $L$-function,

$$
L(s, \rho)=\prod L_{v}(s, \rho)
$$

defined as Euler products convergent for $\operatorname{Re}(s)>>0$ [1]. For most places $v$ (unramified for $\rho$ ) we have

$$
L_{v}(s, \rho)=\operatorname{det}\left(1-\rho\left(F r o b_{v}\right) q_{v}^{-s}\right)^{-1}
$$


is a degree $n$ Euler factor. For $n=1$, these $L(s, \rho)$ figured prominently in Artin's analytic proof of class field theory, so they are quite useful invariants! Much is known about these global invariants (see Chapter 4 of [8]):

1. They have a meromorphic continuation to the complex plane and satisfy an appropriate functional equation (Brauer, 1947);

2. they are bounded in vertical strips;

3. They are conjectured to be entire if $\rho$ does not contain the trivial representation (the Artin Conjecture).

In other words, for irreducible, non-trivial representations $\rho$ these invariants should be nice and hence the representations should be modular. (Note that the twisting of the $L$-function on the modular side corresponds to taking tensor product of Galois representations.) Thus we are naturally lead to:

Global Langlands Conjecture (naive version). There exist natural bijections between (a) the set of irreducible $n$-dimensional representations $\rho$ of $\mathcal{G}_{k}$ and (b) the set of cuspidal automorphic representations $\pi$ of $G L_{n}(\mathbb{A})$. (Of course, one of the qualities inherent in natural is an equality of the invariants $L(s, \rho)=L(s, \pi)$, along with twisted versions, etc.....)

One thing possible with the modern formulation of the theory of automorphic representations is that one can easily formulate a version of local modularity:

Local Langlands Conjecture (naive version). For a local field $k_{v}$, there exist natural bijections between (a) the set of semi-simple $n$-dimensional representations $\rho_{v}$ of $\mathcal{G}_{k_{v}}$ and (b) the set of irreducible admissible representations $\pi_{v}$ of $G L_{n}\left(k_{v}\right)$, again requiring an equality of the local invariants $L\left(s, \rho_{v}\right)=L\left(s, \pi_{v}\right)$, along with twisted versions, etc....

Note: One can view these bijections in two ways

1. passing information from Automorphic (analytic) $\longrightarrow$ Galois (arithmetic); this is a non-abelian class field theory.

2. passing information from Galois $\longrightarrow$ Automorphic; this is an arithmetic parameterization of local or automorphic representations.

I have used the word naive in these formulations. This is naive because of several issues: 
1. The difference in the topologies of $\mathcal{G}_{\mathbb{Q}}$ and $G L_{n}(\mathbb{C})$ is such that one doesn't pick up enough information about the Galois group from complex representations. One needs to use $\ell$-adic representations:

$$
\rho: \operatorname{Gal}(\bar{k} / k) \rightarrow G L_{n}\left(\overline{\mathbb{Q}}_{\ell}\right) \quad \text { or } \quad \rho: G a l\left(\overline{k_{v}} / k_{v}\right) \rightarrow G L_{n}\left(\overline{\mathbb{Q}}_{\ell}\right) .
$$

with $\ell$ a prime.

2. There are "more" automorphic or admissible representations of $G L_{n}$ than there are $n$-dimensional Galois representations.

Weil dealt with the second issue for $n=1$ by introducing the local and global Weil groups $W_{\mathbb{Q}}$ or $W_{\mathbb{Q}_{v}}$ to substitute for $\mathcal{G}_{\mathbb{Q}}$, etc. [61].

Deligne dealt with the first issue and second issue locally for $n \geq 2$ by introducing the local Weil-Deligne group $W_{\mathbb{Q}_{v}}^{\prime}$ to replace $W_{\mathbb{Q}_{v}}[18]$. So

$$
\begin{aligned}
& \mathcal{G}_{\mathbb{Q}_{v}} \longrightarrow W_{\mathbb{Q}_{v}} \longrightarrow W_{\mathbb{Q}_{v}}^{\prime} \quad \text { locally } \\
& \mathcal{G}_{\mathbb{Q}} \longrightarrow W_{\mathbb{Q}} \longrightarrow ? ? ? \quad \text { globally }
\end{aligned}
$$

Which leaves us only with:

Local Langlands Conjecture. For a local field $k_{v}$, there exist natural bijections between (a) the set of (suitable) semi-simple $n$-dimensional (complex) representations $\rho_{v}$ of $W_{k_{v}}^{\prime}$ and (b) the set of irreducible admissible representations $\pi_{v}$ of $G L_{n}\left(k_{v}\right)$, again requiring an equality of the local invariants $L\left(s, \rho_{v}\right)=L\left(s, \pi_{v}\right)$, along with twisted versions, $\varepsilon$-factors, etc.....

This local modularity is in fact a Theorem due to

- Langlands in the case $k_{v}=\mathbb{R}, \mathbb{C}[44]$;

- Laumon-Rapoport-Stuhler in the case $\operatorname{char}\left(k_{v}\right)=p>0[45]$;

- Harris-Taylor, Henniart in the case $\operatorname{char}\left(k_{v}\right)=0$ [25, 29].

Globally, at least over a number field, there is not a nice analogue of the Weil-Deligne group. (The global Weil-Deligne group $W_{k}^{\prime}$ would essentially be the conjectural Langlands group $\mathcal{L}_{k}$. In finite characteristic, one can use the global Galois group $\mathcal{G}_{k}$ for many purposes - see Lafforgue for example [42].) So, at least in characteristic 0 one has difficulties formulating a Global Langlands Conjecture. However, as a practical matter, one can circumvent this difficulty by appealing to a philosophical Hasse principle or local-global compatibility. 
Let us now ask, as Langlands did, how would we formulate these conjectures for other types of Galois representations or other groups $H$ ? We will primarily be interested in the split classical groups $H=S O_{2 n+1}, S O_{2 n}, S p_{2 n}$. To think about this problem, Langlands introduced dual groups ${ }^{L} H$, essentially the complex analytic groups obtained by dualizing the root data:

\begin{tabular}{|c|c|}
\hline$H$ & ${ }^{L} H$ \\
\hline & \\
$G L_{n}$ & $G L_{n}(\mathbb{C})$ \\
$S O_{2 n+1}$ & $S p_{2 n}(\mathbb{C})$ \\
$S p_{2 n}$ & $S O_{2 n+1}(\mathbb{C})$ \\
$S O_{2 n}$ & $S O_{2 n}(\mathbb{C})$ \\
\hline
\end{tabular}

Then the Local Langlands Conjecture for $H$, as an arithmetic parameterization problem, takes the following form.

Local Langlands Conjecture for $H$ : There exists a surjective map $\left\{\pi_{v}:\right.$ irreducible admissible representations of $\left.H\left(\mathbb{Q}_{v}\right)\right\} \rightarrow\left\{\phi_{v}: W_{\mathbb{Q}_{v}}^{\prime} \rightarrow{ }^{L} H(\mathbb{C})\right.$, admissible\}

with finite fibres such that $L\left(s, \pi_{v}\right)=L\left(s, \phi_{v}\right)$ (among other things).

This would partition the admissible representations of $H\left(\mathbb{Q}_{v}\right)$ into $L$-packets, i.e., finite subsets all having the same $L$-functions.

Known cases:

$-k_{v}=\mathbb{R}, \mathbb{C}$, all $H$ (Langlands [44]);

- $k_{v}$ non-archimedean, and $\pi_{v}$ unramified (Satake [49]);

- $H=G L_{n}, k_{v}$ arbitrary (as above).

Again, a Global Langlands Conjecture for $H$ must be thought of in terms of local-global compatibility.

\section{$2.2 \quad$ Functoriality}

(For more details and references we refer the reader to [5] and to Chapter 11 of [8].) We finally come to Functoriality. It is a manifestation of thinking about 
either a Local Langlands Conjecture or Global Langlands Conjecture as giving an arithmetic parameterization of modular data. To explain in the cases of interest, we begin with

- a split reductive group $H$ over $k$ as above;

- its dual group ${ }^{L} H$;

- a complex analytic homomorphism (L-homomorphism) $u:{ }^{L} H \rightarrow G L_{N}(\mathbb{C})$, which we will take as the natural embedding.

One can view the $L$-homomorphism simply as a vehicle for transferring the arithmetic parameters for the representations.

Local Functoriality: If $\pi_{v}$ is an irreducible admissible representation of $H\left(k_{v}\right)$ then we can obtain an irreducible admissible representation $\Pi_{v}$ of $G L_{N}\left(k_{v}\right)$ by following the diagram

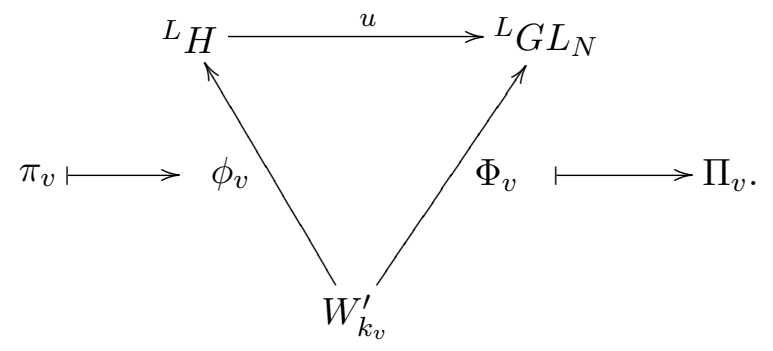

and this should satisfy

$$
L\left(s, \pi_{v}\right)=L\left(s, \phi_{v}\right)=L\left(s, \Phi_{v}\right)=L\left(s, \Pi_{v}\right)
$$

along with similar equalities for twisted versions and for $\varepsilon$-factors.

$\Pi_{v}$ is the local Langlands lift of $\pi_{v}$ to $G L_{N}\left(k_{v}\right)$ (associated to the $L$-homomorphism $u$ ).

In the case of Global Functoriality, since we do not have a global version of the Weil-Deligne group, and so no such global diagram, we rely on local/global compatibility, but with a fixed $L$-homomorphism acting as our "global glue" for the local parameters.

Global Functoriality Conjecture: If $\pi=\otimes^{\prime} \pi_{v}$ is a cuspidal automorphic representation of $H(\mathbb{A})$ then the representation $\Pi=\otimes^{\prime} \Pi_{v}$ of $G L_{N}(\mathbb{A})$ we obtain 
by following the diagram

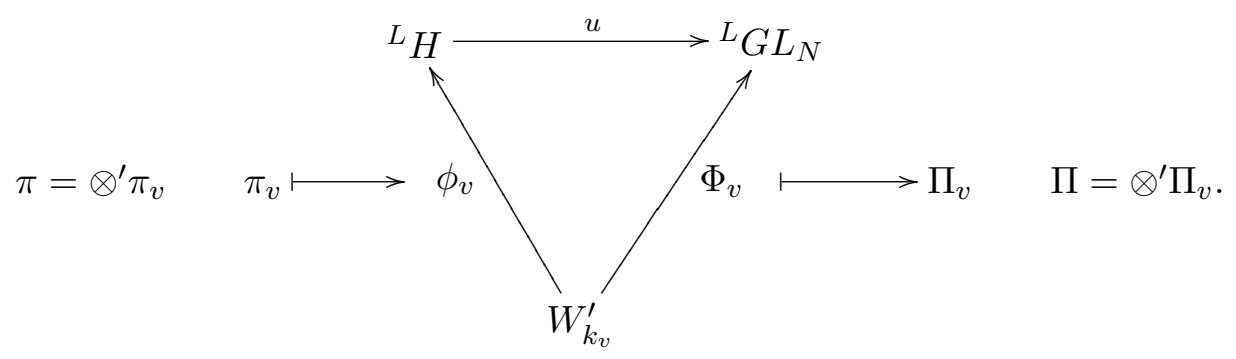

should be automorphic and moreover should satisfy

$$
L(s, \pi)=\prod_{v} L\left(s, \pi_{v}\right)=\prod_{v} L\left(s, \Pi_{v}\right)=L(s, \Pi)
$$

along with similar equalities for twisted versions and for $\varepsilon$-factors.

The global lifting $\pi \rightarrow \Pi$, which relates $L$-functions, is a purely automorphic manifestation of the presumed modularity of global Galois representations!

\subsection{Converse Theorem}

Let us return to thinking about how the Converse Theorem is related to questions of modularity and thereby Functoriality. It is made to order for Global Functoriality. Here is the idea.

Take a global field $k$ and a split reductive algebraic group $H$ over $k$. Let $u:{ }^{L} H \rightarrow G L_{N}(\mathbb{C})$ be the $L$-homomorphism as before and $\pi=\otimes_{v}^{\prime} \pi_{v}$ a cuspidal automorphic representations of $H(\mathbb{A})$.

Step 1: We apply the Local Langlands Conjecture to each $\pi_{v}$ as above to produce a local representation $\Pi_{v}$ of $G L_{N}\left(k_{v}\right)$ and then put them together to form a candidate lift $\Pi=\otimes_{v}^{\prime} \Pi_{v}$ as an irreducible admissible representation of $G L_{N}(\mathbb{A})$.

Step 2. Since the Local Langlands Conjecture entails the matching of analytic invariants, then for all cuspidal $\pi^{\prime}$ of $G L_{m}(\mathbb{A}), 1 \leq m \leq N-2$ we expect that

$$
L\left(s, \Pi \times \pi^{\prime}\right)=L\left(s, \pi \times \pi^{\prime}\right)
$$

etc... Now the $L$-functions on the right hand side are automorphic associated to $H(\mathbb{A}) \times G L_{m}(\mathbb{A})$ and so should be verifiably nice!

Step 3. We now apply the Converse Theorem to conclude that $\Pi$ is automorphic, i.e., establishing Global Functoriality from $H$ to $G L_{N}$.

There are two immediate problems that come to mind: 
1. the lack of the Local Langlands Conjecture for $H$ at all places $v$;

2. the actual analytic control of the $L$-functions $L\left(s, \pi \times \pi^{\prime}\right)$ for $H$.

When have we been able to either solve or circumvent these problems?

\subsection{Examples of established Global Functorialities}

We let $k$ be a number field, $\pi$ a globally generic cuspidal representation of $H(\mathbb{A})$. Then the above method, or a variant of it, has been used to establish a version of global functoriality in the following cases:

\begin{tabular}{|c|c|c|c|c|c|}
\hline$H$ & ${ }^{L} H$ & $u:{ }^{L} H \rightarrow{ }^{L} G$ & ${ }^{L} G$ & $G$ & \\
\hline$G L_{2} \times G L_{2}$ & $G L_{2}(\mathbb{C}) \times G L_{2}(\mathbb{C})$ & $\otimes$ & $G L_{4}(\mathbb{C})$ & $G L_{4}$ & {$[47]$} \\
\hline$S O_{2 n+1}$ & $S p_{2 n}(\mathbb{C})$ & $\hookrightarrow$ & $G L_{2 n}(\mathbb{C})$ & $G L_{2 n}$ & {$[11,12]$} \\
\hline $\mathrm{SO}_{2 n}$ & $S O_{2 n}(\mathbb{C})$ & $\hookrightarrow$ & $G L_{2 n}(\mathbb{C})$ & $G L_{2 n}$ & {$[12]$} \\
\hline$S p_{2 n}$ & $S O_{2 n+1}(\mathbb{C})$ & $\hookrightarrow$ & $G L_{2 n+1}(\mathbb{C})$ & $G L_{2 n+1}$ & {$[12]$} \\
\hline$G L_{2} \times G L_{3}$ & $G L_{2}(\mathbb{C}) \times G L_{3}(\mathbb{C})$ & $\otimes$ & $G L_{6}(\mathbb{C})$ & $G L_{6}$ & {$[39]$} \\
\hline$G L_{4}$ & $G L_{4}(\mathbb{C})$ & $\wedge^{2}$ & $G L_{6}(\mathbb{C})$ & $G L_{6}$ & {$[37]$} \\
\hline$G L_{2} / E$ & & Asai & & $G L_{4}$ & {$[41,48]$} \\
\hline$U_{n, n}$ & & Base Change & & $G L_{2 n} / E$ & {$[38]$} \\
\hline $\operatorname{GSpin}_{2 n+1}$ & $G S p_{2 n}(\mathbb{C})$ & $\hookrightarrow$ & $G L_{2 n}(\mathbb{C})$ & $G L_{2 n}$ & {$[2]$} \\
\hline GSpin $_{2 n}$ & $G S O_{2 n}(\mathbb{C})$ & $\hookrightarrow$ & $G L_{2 n}(\mathbb{C})$ & $G L_{2 n}$ & {$[2]$} \\
\hline
\end{tabular}

Remarks. The major difficulty in establishing these results has been the control of the twisted $L$-functions $L\left(s, \pi \times \pi^{\prime}\right)$ for $H \times G L_{m}$. In almost all cases here, these $L$-functions were controlled by the Langlands-Shahidi method which involves realizing the $L$-functions in the constant and non-constant Fourier coefficients of Eisenstein series (see [50] and the references therein). Recently Shahidi 
with crucial collaboration of Kim have shown that the $L\left(s, \pi \times \pi^{\prime}\right)$ are nice in the above situations. This method accounts for the restriction to globally generic representations (so that we have suitable Fourier coefficients), the restriction to number fields (a historical restriction, not an essential restriction of the method) and the finiteness of the table (an honest restriction of the method).

In actuality, in having to circumvent the lack of the Local Langlands Conjecture and some remaining global problems, one loses control of $\Pi$ at a finite set of finite places. The true conclusion is: there exists an automorphic representation $\Pi=\otimes_{v}^{\prime} \Pi_{v}$ such that $\Pi_{v}$ is the local Langlands lift of $\pi_{v}$ at all but finitely many places. After this, one has to do further work to remove this ambiguity, deal with cuspidality, characterize the image, etc.. But these questions no longer involve the Converse Theorem in an essential way.

Question. Other than indirect evidence for the Global Langlands Conjecture and hence the global modularity of Galois representations, are these results applicable to any "practical" problems of number theory? So we ask again: who cares?

\section{Applications}

As Arthur noted in his Whittemore Lectures, establishing liftings from classical groups to $G L_{n}$ can yield facts for the classical groups by pulling back facts from $G L_{n}$. Two examples of these types of results that resulted from these liftings (plus a lot of work) were

- estimates towards the Ramanujan conjecture for generic cuspidal representations of split classical groups $[12,51]$;

- the Local Langlands Conjecture for generic representations of $S O_{2 n+1}\left(k_{v}\right)$ for a $p$-adic local field by Jiang and Soudry [35, 36].

But here I want to primarily address applications to two questions of "classical" number theory.

\subsection{The Ramanujan Conjecture}

[24] Ramanujan was originally interested in the behavior of an arithmetical function $\tau(n)$ defined as the coefficient of $x^{n}$ in the product

$$
x\left(\prod_{n=1}^{\infty}\left(1-x^{n}\right)\right)^{24}=\sum_{n=1}^{\infty} \tau(n) x^{n} .
$$


Presumably based on numerical calculations he conjectured in 1916 that for prime $p$

$$
\tau(p) \leq 2 p^{11 / 2} .
$$

If we replace $x$ by $q=e^{2 \pi i z}$ we obtain the Jacobi product expansion of the unique cusp form of weight 12 for $S L_{2}(\mathbb{Z})$

$$
e^{2 \pi i z}\left(\prod_{n=1}^{\infty}\left(1-e^{2 \pi i n z}\right)\right)^{24}=\sum_{n=1}^{\infty} \tau(n) e^{2 \pi i n z}=\Delta(z)
$$

the discriminant function from the theory of elliptic modular forms. Since the dimension of the space of cusp forms of weight 12 for $S L_{2}(\mathbb{Z})$ is one, $\Delta(z)$ must be a Hecke eigenform and

$$
L(s, \Delta)=\sum_{n=1}^{\infty} \frac{\tau(n)}{n^{s}}=\prod_{p}\left(1-\tau(p) p^{-s}+p^{11} p^{-2 s}\right)^{-1} .
$$

Over time this conjecture was generalized by Petersson to other normalized Hecke eigenforms. If we have

$$
f(z)=\sum_{n=1}^{\infty} a_{n} e^{2 \pi i n z} \in S_{k}(\Gamma)
$$

a Hecke eigenform normalized so that $a_{1}=1$ with

$$
L(s, f)=\sum_{n=1}^{\infty} \frac{a_{n}}{n^{s}}=\prod_{p}\left(1-a_{p} p^{-s}+p^{k-1} p^{-2 s}\right)^{-1}
$$

then the Ramanujan-Petersson conjecture is that

$$
\left|a_{p}\right| \leq 2 p^{\frac{k-1}{2}} .
$$

Of course, there is also a version for Maaß forms.

- For classical holomorphic modular forms (over $\mathbb{Q}$ ) this is now a Theorem due to Shimura $(k=2)$, Deligne $(k \geq 2)$, and Deligne-Serre $(k=1)$ $[53,17,19,20]$.

- For Maaß forms this is still open.

- For forms over number fields this is open as far as I know (with the exception of some results for holomorphic Hilbert modular forms [7, 4]). 
In the modern formulation, if the Hecke eigenform $f(z)$ of weight $k$ is associated to the cuspidal automorphic representation $\pi=\otimes^{\prime} \pi_{v}$ of $G L_{2}(\mathbb{A})$, then

$$
\Lambda\left(s+\frac{k-1}{2}, f\right)=L(s, \pi)
$$

and for primes not dividing the level of $f(z)$ (all primes for $\Gamma=S L_{2}(\mathbb{Z})$ ) we have

$$
\left(1-\frac{a_{p}}{p^{(k-1) / 2}} p^{-s}+p^{-2 s}\right)^{-1}=L\left(s, \pi_{p}\right) .
$$

Now let us return to the Local Langlands Conjecture for $G L_{2}\left(\mathbb{Q}_{p}\right)$. If $\pi_{p}$ is unramified (so $p$ not dividing the level of $f$ and $\pi_{p}$ having a $G L_{2}\left(\mathbb{Z}_{p}\right)$ fixed vector) then the corresponding parameter

$$
\pi_{p} \mapsto\left[\phi_{p}: W_{\mathbb{Q}_{p}}^{\prime} \rightarrow G L_{2}(\mathbb{C})\right]
$$

is also unramified (so trivial on the inertia subgroup) and is determined by the image of the Frobenius at $p, \phi_{p}\left(F_{r o b}\right)$, which is a semi-simple element (actually conjugacy class) of $G L_{2}(\mathbb{C})$

$$
\phi_{p}\left(F r o b_{p}\right)=\left(\begin{array}{cc}
\alpha_{p} & 0 \\
0 & \beta_{p}
\end{array}\right)=A_{\pi_{p}} \in G L_{2}(\mathbb{C}),
$$

the Hecke-Satake parameters of $\pi_{p}$, and

$$
L\left(s, \pi_{p}\right)=\operatorname{det}\left(1-\phi_{p}\left(\text { Frob }_{p}\right) p^{-s}\right)^{-1}=\left[\left(1-\alpha_{p} p^{-s}\right)\left(1-\beta_{p} p^{-s}\right)\right]^{-1} .
$$

Comparing the two expressions for $L\left(s, \pi_{p}\right)$ we find

$$
\operatorname{tr}\left(A_{\pi_{p}}\right)=\alpha_{p}+\beta_{p}=\frac{a_{p}}{p^{(k-1) / 2}} \quad \text { and } \quad \operatorname{det}\left(A_{\pi_{p}}\right)=\alpha_{p} \beta_{p}=1 .
$$

Thus the Ramanujan-Petersson conjecture translates into

$$
\left|\alpha_{p}+\beta_{p}\right|=\left|\alpha_{p}+\alpha_{p}^{-1}\right| \leq 2 \quad \text { or } \quad\left|\alpha_{p}\right|=\left|\beta_{p}\right|=1 .
$$

This has an immediate generalization to any global field.

Generalized Ramanujan Conjecture for $G L_{2}$ : If $\pi$ is a cuspidal automorphic representation of $G L_{2}\left(\mathbb{A}_{k}\right), \pi=\otimes^{\prime} \pi_{v}$, and $v$ is a finite place for which $\pi_{v}$ is unramified, then the Hecke-Satake parameters at $v$ satisfy $\left|\alpha_{v}\right|=1$.

Similarly, if $\pi=\otimes^{\prime} \pi_{v}$ is a cuspidal automorphic representation of $G L_{n}(\mathbb{A})$ then as before if $\pi_{v}$ is unramified then

$$
\pi_{v} \mapsto\left[\phi_{v}: W_{k_{v}}^{\prime} \rightarrow G L_{n}(\mathbb{C})\right] \quad \text { and } \quad \phi_{v}\left(\operatorname{Frob}_{v}\right)=\left(\begin{array}{ccc}
\alpha_{1, v} & & \\
& \ddots & \\
& & \alpha_{n, v}
\end{array}\right)
$$


and the Generalized Ramanujan Conjecture for $G L_{n}$, in its most elementary form, says $\left|\alpha_{i, v}\right|=1$ for all $i$ and all unramified places $v$.

Remark: From the local theory of integral representation, Jacquet and Shalika were able to establish at least a uniform estimate for all $G L_{n}[9]$, namely if at the unramified place $v$ we have $k_{v} \supset \mathfrak{o}_{v} \supset \mathfrak{p}_{v}$ with $\left|\mathfrak{o}_{v} / \mathfrak{p}_{v}\right|=q_{v}$ then

$$
q_{v}^{-1 / 2}<\left|\alpha_{i, v}\right|<q_{v}^{1 / 2} \text {. }
$$

How does this relate to our lifts? First, a bit more Functoriality. From the lifts

\begin{tabular}{|c|c|c|c|c|}
\hline$H$ & ${ }^{L} H$ & $u:{ }^{L} H \rightarrow{ }^{L} G$ & ${ }^{L} G$ & $G$ \\
\hline$G L_{2} \times G L_{3}$ & $G L_{2}(\mathbb{C}) \times G L_{3}(\mathbb{C})$ & $\otimes$ & $G L_{6}(\mathbb{C})$ & $G L_{6}$ \\
$G L_{4}$ & $G L_{4}(\mathbb{C})$ & $\wedge^{2}$ & $G L_{6}(\mathbb{C})$ & $G L_{6}$ \\
\hline
\end{tabular}

described earlier using the Converse Theorem, Kim and Shahidi [39, 37] were able to deduce the lifts

\begin{tabular}{|c|c|c|c|c|}
\hline$H$ & ${ }^{L} H$ & $u:{ }^{L} H \rightarrow{ }^{L} G$ & ${ }^{L} G$ & $G$ \\
\hline$G L_{2}$ & $G L_{2}(\mathbb{C})$ & $S_{y m}$ & $G L_{4}(\mathbb{C})$ & $G L_{4}$ \\
$G L_{2}$ & $G L_{2}(\mathbb{C})$ & Sym $^{4}$ & $G L_{5}(\mathbb{C})$ & $G L_{5}$ \\
\hline
\end{tabular}

On the level of parameters, this corresponds to the diagrams

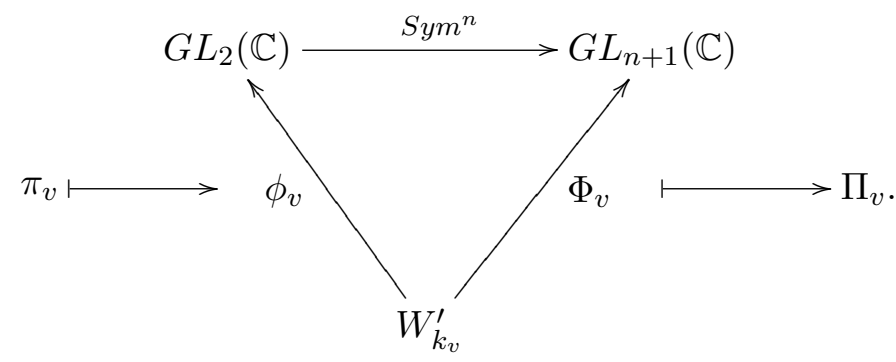

for $n=3,4$. So if $\pi \simeq \otimes^{\prime} \pi_{v}$ is a cuspidal representation of $G L_{2}(\mathbb{A})$ then at the unramified places $v$ the Hecke-Satake parameters are related as follows: if

$$
\phi_{v}\left(F r o b_{v}\right)=A_{\pi_{v}}=\left(\begin{array}{cc}
\alpha_{1, v} & \\
& \alpha_{2, v}
\end{array}\right)
$$


then

$$
\Phi_{v}\left(F r o b_{v}\right)=A_{\Pi_{v}}=\left(\begin{array}{ccccc}
\alpha_{1, v}^{n} & & & \\
& \alpha_{1, v}^{n-1} \alpha_{2, v} & & & \\
& & \ddots & \\
& & & \alpha_{1, v} \alpha_{2, v}^{n-1} & \\
& & & & \alpha_{2, v}^{n}
\end{array}\right) .
$$

So if we simply apply the Jacquet-Shalika bounds to the lift $\Pi_{v}$ we obtain

$$
q_{v}^{-1 / 2}<\left|\alpha_{i, v}^{n}\right|<q_{v}^{1 / 2} \quad \text { or } \quad q_{v}^{-1 / 2 n}<\left|\alpha_{i, v}\right|<q_{v}^{1 / 2 n} .
$$

So, as Langlands pointed out: the symmetric power functorialities for all $n$ implies the full Ramanujan conjecture for $G L_{2}$.

The $S y m^{4}$ functoriality of Kim and Shahidi gives a non-trivial bound towards Ramanujan for $G L_{2}$ of

$$
q_{v}^{-1 / 8}<\left|\alpha_{i, v}\right|<q_{v}^{1 / 8} .
$$

(In fact, by analyzing the behavior of the symmetric power $L$-functions $L\left(s, \pi, S m^{n}\right)$ for $n \leq 9$, Kim and Shahidi [40] manage to deduce the improved bound

$$
q_{v}^{-1 / 9}<\left|\alpha_{i, v}\right|<q_{v}^{1 / 9}
$$

even over a number field!)

Note 1: This gives estimates for Fourier coefficients of classical Maaß forms or general modular forms for $G L_{2}$ over number fields [40, 51].

Note 2: At the archimedean places there are similar results which for Maaß forms give lower bounds for the first eigenvalue of the Laplacian (i.e. Selberg's Conjecture) of $\lambda_{1} \geq \frac{77}{324}[40,52]$.

We will return to this story in a minute, but now we turn to our second application.

\subsection{Hilbert's Eleventh Problem}

[30] This is the problem of understanding integral quadratic forms over number fields.

Example: Let $K$ be a totally real number field, $\mathfrak{o}$ its ring of integers, and $\alpha \in \mathfrak{o}$. When can we write

$$
\alpha=x_{1}^{2}+x_{2}^{2}+x_{3}^{2} \quad \text { with } \quad x_{i} \in \mathfrak{o}
$$


and how often? More generally, if

$$
q(\vec{x})=\sum_{i, j=1}^{3} a_{i, j} x_{i} x_{j} \quad \text { with } \quad a_{i, j} \in \mathfrak{o}
$$

is a positive definite integral ternary quadratic form, when can we write $\alpha=q(\vec{x})$ with $\vec{x} \in \mathfrak{o}^{3}$ ?

This is seemingly unrelated to what we have been discussing. But as anyone who has studied Siegel knows, if $(K: \mathbb{Q})=d$ and we take $\tau \in \mathfrak{H}^{d}$ and form the generating function

$$
\vartheta_{q}(\tau)=\sum_{\vec{x} \in \mathfrak{o}^{3}} e^{2 \pi i \operatorname{tr}(q(\vec{x}) \tau)}=1+\sum_{\alpha \in \mathfrak{o}} r_{q}(\alpha) e^{2 \pi i \operatorname{tr}(\alpha \tau)}
$$

then this is a Hilbert modular form of weight $3 / 2$ for a congruence subgroup $\Gamma \subset S L_{2}(\mathfrak{o})$ [56]. Thus the representation numbers

$$
r_{q}(\alpha)=\left|\left\{\vec{x} \in \mathfrak{o}^{3} \mid \alpha=q(\vec{x})\right\}\right|
$$

are the Fourier coefficients of a modular form. (As Eichler said, ......).

Note: This is another type of modularity: integral quadratic forms $q(\vec{x})$ correspond to theta series $\vartheta_{q}(\tau)$, which Siegel called analytic class invariants.

Thanks to the work of Waldspurger $(K=\mathbb{Q})$ and Shimura and Baruch-Mao ( $K$ totally real) $[60,54,3]$ the square free Fourier coefficients, call them $a(\alpha)$ of a $3 / 2$ weight cusp form $\tilde{f}$ are controlled by the $L$-function of a weight 2 companion cusp form $\varphi$ (related by the Shimura correspondence). Through this relation, controlling the $r_{q}(\alpha)$ comes down to controlling the special values $L\left(\frac{1}{2}, \varphi, \chi_{\alpha}\right)$ as $\alpha$ varies, where $\chi_{\alpha}$ is the quadratic character of $K$ associated to the extension $K(\sqrt{\alpha}) / K$ by class field theory and $\varphi \in S_{2}\left(\Gamma^{\prime}\right)$.

It is usually easy to get a weak estimate on $L$-functions, called the convexity bound (due to its relation with the Phragmen-Lindelöf convexity principle), which in this case is

$$
\left|L\left(\frac{1}{2}, \varphi, \chi_{\alpha}\right)\right|<<N(\alpha)^{\frac{1}{2}+\epsilon}
$$

and as a general principle, breaking convexity can have substantial consequences. Recently with Piatetski-Shapiro and Sarnak we broke convexity here to obtain

$$
\left|L\left(\frac{1}{2}, \varphi, \chi_{\alpha}\right)\right|<<N(\alpha)^{\frac{1}{2}-\frac{7}{130}+\epsilon}
$$

for a weight two Hilbert modular cusp form $\varphi$. The proof of this would take us too far afield. However, what is important for us here, is that the number $\frac{7}{130}$ by 
which we break convexity relies on the use of the $\frac{1}{9}$ bounds towards RamanujanPetersson for Hilbert modular Maaß form of Kim and Shahidi discussed above in a crucial way, i.e, an application of Functoriality. This (and in fact any) breaking of convexity then yields:

Theorem [10, 16] Let $K$ be a totally real field, $q$ a positive definite integral ternary quadratic form. The there exists an (ineffective) constant $C$ such that for all $\alpha \in \mathfrak{o}$, square free, totally positive and such that $N(\alpha)>C$ we can solve

$$
\alpha=q(\vec{x}) \quad \text { with } \quad \vec{x} \in \mathfrak{o}^{3}
$$

iff

$$
\alpha=q\left(\overrightarrow{x_{\mathfrak{p}}}\right) \quad \text { with } \quad \overrightarrow{x_{\mathfrak{p}}} \in \mathfrak{o}_{\mathfrak{p}}^{3} \quad \text { locally for all } \quad \mathfrak{p} \text {. }
$$

The question of local representability was solved definitively and quantitatively by Siegel in the 1930's [55]. This case of positive definite ternary forms over a totally real field was the last remaining case of Hilbert's eleventh problem from the 1900 ICM [30].

\subsection{A return to modularity}

There have been two spectacular cases of modularity established recently:

1. Every elliptic curve over $\mathbb{Q}$ is modular, due to Wiles, Taylor, Breuil, Conrad and Diamond, as discussed above $[63,6]$.

2. The Global Langlands Conjecture for $G L_{n}$ over a function field over a finite field, so the modularity of global $\ell$-adic Galois representations, by Lafforgue [42].

It is a fair question to ask whether the Converse Theorems played any role in these results. The answer is that in each case the Converse Theorems did play a supporting role.

In Wiles' work to establish modularity in general, he roughly need to know one specific case of modularity to feed in to his deformation theory. He obtained what he needed from the work of Langlands and Tunnell on the modularity of tetrahedral and octahedral Galois representations [43, 59]. Each of these results were an application of base change for $G L_{2}$, but again they needed something to be modular to give them a starting point. For Langlands this was the modularity of three dimensional dihedral Galois representations which had been established 
by Jacquet, Piatetski-Shapiro and Shalika with the Converse Theorem for $G L_{3}$ [32]. Then Tunnell began with the result of Langlands and then needed in addition the existence of non-normal cubic base change, again a consequence of the Converse Theorem for $G L_{3}$ [33]. I believe that at this point in time, both of these results can be established by alternate methods, but at the time the Converse Theorem was the only known way of obtaining these results.

In the work of Lafforgue the primary technical tool was the Arthur-Selberg trace formula. However, one aspect of his proof was inductive. The key to this induction is what Lafforgue refers to as the "principe de récurrence de Deligne" (sometimes credited to Deligne and Piatetski-Shapiro) which had as one key ingredient the Converse Theorem for $G L_{n}$ for function fields as in PiatetskiShapiro's early preprint [46]. The Converse Theorems that we have discussed above were formulated in the analytic language that is most appropriate for questions over number fields. For function fields, it is best to have a formulation in the language of rational functions. The best reference for such a formulation is in fact Appendice B of Lafforgue's paper [42].

So indeed the Converse Theorem played a small supporting role in both these questions of modularity.

\section{References}

[1] E. Artin, Über eine neue Art von L-Reihen. Hamb. Abh. (1923), 89-108.

[2] M. Asgari and F. Shahidi, Generic transfer for general spin groups. Duke Math. J., to appear.

[3] M. Baruch and Z. Mao, Central value of automorphic L-functions. Preprint (2000).

[4] D. Blasius, Hilbert modular forms and the Ramanujan conjecture. Preprint (2004)

[5] A. Borel, Automorphic L-functions. Proc. Symp. Pure Math. 33, Part 2, (1979), 27-61.

[6] C. Breuil, B. Conrad, F. Diamond and R. Taylor. On the modularity of elliptic curves over $\mathbb{Q}$. J. Amer. Math. Soc. 14 (2001), 843-939.

[7] J-L. Brylinski and J-P. Labesse, Cohomologie d'intersection et fonctions L de certaines variétiés de Shimura. Ann. Sci. Éc. Norm. Sup. (4) 19 (1986), 361-412. 
[8] D. Bump, et al. An Introduction to the Langlands Program (J. Bernstein and S. Gelbart, editors). Birkhäuser, Boston-Basel-Berlin, 2003.

[9] J.W. Cogdell, L-functions and Converse Theorems for $G L_{n}$. Automorphic Forms and Applications, IAS/Park City Mathematics Series, to appear.

[10] J.W. Cogdell, On sums of three squares. J. Théor. Nombres Bordeaux 15 (2003), 33-44.

[11] J.W. Cogdell, H.H. Kim, I.I. Piatetski-Shapiro, and F. Shahidi, On lifting from classical groups to $G L_{N}$. Publ. Math. IHES 79 (2001), 5-30.

[12] J.W. Cogdell, H.H. Kim, I.I. Piatetski-Shapiro, and F. Shahidi, Functoriality for the classical groups, Publ. Math. IHES 99 (2004), 163-233.

[13] J.W. Cogdell and I.I. Piatetski-Shapiro, Converse Theorems for GL $L_{n}$. Publ. Math. IHES 79 (1994), 157-214.

[14] J.W. Cogdell and I.I. Piatetski-Shapiro, Converse Theorems for $G L_{n}$, II. J. reine angew. Math. 507 (1999), 165-188.

[15] J.W. Cogdell and I.I. Piatetski-Shapiro, Remarks on Rankin-Selberg convolutions. Contributions to Automorphic forms, geometry, and Number Theory, 255-278. Johns Hopkins Univ. Press, Baltimore, MD, 2004.

[16] J.W. Cogdell, I.I. Piatetski-Shapiro, and P. Sarnak, Estimates on the critical line for Hilbert modular L-functions and applications, in preparation.

[17] P. Deligne, Forms modulaires et représentations $\ell$-adiques. Séminaire Bourbaki, vol. 1968/1969, exposé no. 355, Lecture Notes 179 , Springer-Verlag, 1971, 139-172.

[18] P. Deligne, Formes modulaires et représentations de GL(2). Modular Forms of One Variable II, Lecture Notes in mathematics No.349, Springer-Verlag, 1973, 55-105.

[19] P. Deligne, La conjecture de Weil. I. Publ. Math IHES 43 (1974), 273-307.

[20] P. Deligne and J-P. Serre, Formes modulaires de poids 1. Ann. Sci. Éc. Norm. Sup. 7 (1974) 507-530

[21] S. Gelbart and F. Shahidi, Boundedness of automorphic L-functions in vertical strips. J. Amer. Math. Soc. 14 (2001), 79-107.

[22] I.M. Gelfand and I.I. Piatetski-Shapiro, Automorphic functions and representation theory. Trudy Moskov. Mat. Obsc. 12 (1963), 389-412. [Trans. Moscow Math. Soc. 12 (1963), 438-464.] 
[23] H. Hamburger, Über die Riemannsche Funktionalgleichung der $\zeta$-funktion. Math. Zeit. 10 (1921), 240-254; 11 (1922), 224-245; 13 (1922), 283-311.

[24] G.H. Hardy, Ramanujan. AMS Chelsea Publishing, Providence,1999.

[25] M. Harris and R. Taylor, The geometry and cohomology of certain simple Shimura varieties. Annals of Math Studies 151, Princeton University Press, 2001.

[26] E. Hecke, Über die Bistimmung Dirichletscher Reihen durch ihre Funktionalgleichung. Math. Ann. 112 (1936), 664-699.

[27] E. Hecke, Lectures on Dirichlet Series, Modular Functions and Quadratic Forms (edited by Bruno Schoeneberg). Vandenhoeck \& Ruprecht, Göttingen, 1983.

[28] E. Hecke, Mathematische Werke. Vandenhoeck \& Ruprecht, Göttingen, 1983.

[29] G. Henniart, Une preuve simple des correspondance de Langlands pour $G L(n)$ sur un corps p-adic. Invent. math. 139 (2000), 439-455.

[30] D. Hilbert, Mathematical problems. Bull. AMS 8 (1902), 437-479.

[31] H. Jacquet and R.P. Langlands, Automorphic Forms on $G L(2)$. Springer Lecture Notes in Mathematics No.114, Springer Verlag, Berlin, 1970.

[32] H. Jacquet, I.I. Piatetski-Shapiro and J. Shalika, Automorphic forms on GL(3). Ann. of Math. (2) 109 (1979), 169-258.

[33] H. Jacquet, I.I. Piatetski-Shapiro and J. Shalika, Relèvement cubique non normal. C. R. Acad. Sci. Paris Sér. I Math. 292 (1981), no. 12, 567-571.

[34] H. Jacquet, I.I. Piatetski-Shapiro and J. Shalika, Rankin-Selberg convolutions. Amer. J. Math. 105 (1983), 367-464.

[35] D. Jiang and D. Soudry, The local converse theorem for $S O(2 n+1)$ and applications. Ann. of Math. 157 (2003), 743-806.

[36] D. Jiang and D. Soudry, Generic representations and local Langlands reciprocity law for p-adic $\mathrm{SO}_{2 n+1}$. Contributions to Automorphic Forms, Geometry and Number Theory (H. Hida, D. Ramakrishnan, and F. Shahidi, eds.), Johns Hopkins University Press, Baltimore, 2004, 457-519.

[37] H. Kim, Functoriality for the exterior square of $G L_{4}$ and the symmetric fourth of $G L_{2}$. J. Amer. Math. Soc. 16 (2003), 139-183. 
[38] H. Kim and M. Krishnamurthy, Stable base change from unitary groups to $G L_{N}$. IMRN, to appear.

[39] H. Kim and F. Shahidi, Functorial products for $G L_{2} \times G L_{3}$ and the symmetric cube for $G L_{2}$. Ann. of Math. 155 (2002), 837-893.

[40] H. Kim and F. Shahidi, Cuspidality of symmetric powers with applications. Duke Math. J., 112 (2002), 177-197.

[41] M. Krishnamurthy, The Asai transfer to $\mathrm{GL}_{4}$ via the Langlands-Shahidi method. IMRN 2003, no. 41, 2221-2254.

[42] L. Lafforgue, Chtoucas de Drinfled et correspondance de Langlands. Invent. Math. 147 (2002), 1-241.

[43] R.P. Langlands, Base Change for $G L(2)$. Annals of Math. Studies No.96. Princeton University Press, Princeton, 1980.

[44] R.P. Langlands, On the classification of irreducible representations of real algebraic groups. Representation Theory and Harmonic Analysis on Semisimple Lie Groups, Mathematical Surveys and Monographs, No.13, AMS, Providence, 1989, 101-170.

[45] G. Laumon, M. Rapoport, and U. Stuhler, D-elliptic sheaves and the Langlands correspondence. Invent. math. 113 (1993), 217-338.

[46] I.I. Piatetski-Shapiro, Zeta-functions of $G L(n)$. Preprint, University of Maryland (1976).

[47] D. Ramakrishnan, Modularity of the Rankin-Selberg L-series, and multiplicity one for SL(2). Ann. of Math. (2) 152 (2000), 45-111.

[48] D. Ramakrishnan, Modularity of solvable Artin representations of $\mathrm{GO}(4)$ type. IMRN 2002, no. 1, 1-54.

[49] I. Satake, Theory of spherical functions on reductive algebraic groups over a $\mathfrak{p}$-adic field. Publ. Math. IHES 18 (1963), 5-70.

[50] F. Shahidi, Automorphic L-functions and functoriality. Proceedings of the International Congress of Mathematicians, Vol. II (Beijing, 2002). Higher Ed. Press, Beijing, 2002, 655-666.

[51] F. Shahidi, On the Ramanujan conjecture for quasisplit groups. Asian Journal of Math. 8 (2004), 813-836. 
[52] F. Shahidi, Functoriality and small eigenvalues of the Laplacian on Riemann surfaces. Surveys in Differential Geometry, to appear.

[53] G. Shimura, Sur les intégrales attachées aux formes automorphes. J. Math. Soc. Japan 11 (1959), 291-311.

[54] G. Shimura, On the Fourier coefficients of Hilbert modular forms of halfintegral weight. Duke Math. J. 72 (1993), 501-557.

[55] C.L. Siegel, Über die analytische Theorie der quadratischer Formen I. Ann. Math 36 (1935), 527-606; II, 37 (1936), 230-263; III 38 (1937), 212-291.

[56] C.L. Siegel, Gesammelte Abhandlungen. Springer-Verlag, Berlin-HeidelbergNew York, 1966.

[57] R. Taylor, Galois representations. Proceedings of the International Congress of Mathematicians, Vol. I (Beijing, 2002). Higher Ed. Press, Beijing, 2002, $449-474$.

[58] R. Taylor, Galois representations. Ann. Fac. Sci. Toulouse Math. 13 (2004), no. $1,73-119$

[59] J. Tunnell, Artin's conjecture for representations of octahedral type. Bull. Amer. Math. Soc. (N.S.) 5 (1981), 173-175.

[60] J-L. Waldspurger, Sur les coefficients de Fourier des formes modulaires de poids demi-entier. J. Math. Pures Appl. 60 (1981), 375-484.

[61] A. Weil, Sur la théorie du corps de classes. J. Math. Soc. Japan 3 (1951), $1-35$.

[62] A. Weil, Über die Bistimmung Dirichletscher Reihen durch Funktionalgleichungen. Math. Ann. 168 (1967), 149-156.

[63] A. Wiles, Modular elliptic curves and Fermat's last theorem. Annals of Math. 141 (1995), 443-551.

[64] A. Wiles, The Birch and Swinnerton-Dyer Conjecture. www.claymath.org/millennium/Birch_and_SwinnertonDyer_Conjecture/BSD.pdf.

J.W. Cogdell

Department of Mathematics

Ohio State University

Columbus, OH 43210

E-mail:cogdell@math.ohio-state.edu 